\title{
Assessing the Role of Framework Geology on Barrier Island Geomorphology
}

\author{
Phillipe Wernette ${ }^{1 *}$, Chris Houser ${ }^{2}$, Bradley Weymer ${ }^{3}$, Mark Everett ${ }^{4}$, \\ Michael P. Bishop ${ }^{1}$, and Bobby Reece ${ }^{4}$ \\ ${ }^{1}$ Texas A\&M University, Department of Geography, College Station, TX, USA \\ 2University of Windsor, Department of Earth and Environmental Sciences, Windsor, ON, Canada \\ ${ }^{3}$ GEOMAR Helmholtz Center for Ocean Research Kiel, Kiel, Germany \\ ${ }^{4}$ Texas A\&M University, Department of Geology and Geophysics, College Station, TX, USA
}




\section{Coastal Geomorphology}

"It is essential to understand this geologic framework before attempting to model the large-scale behavior of these types of coastal systems."

(Riggs et al, 1995)

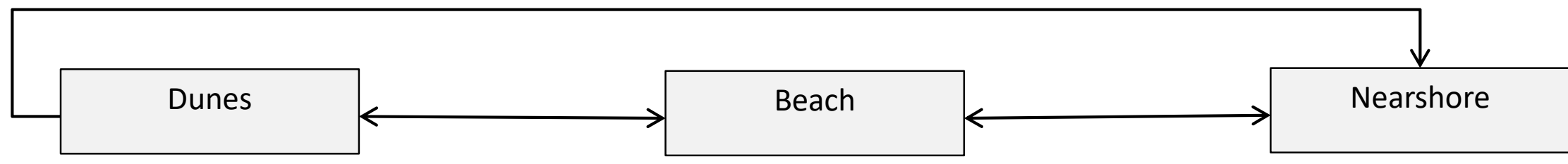

Geologic Framework

Storm-impact Model

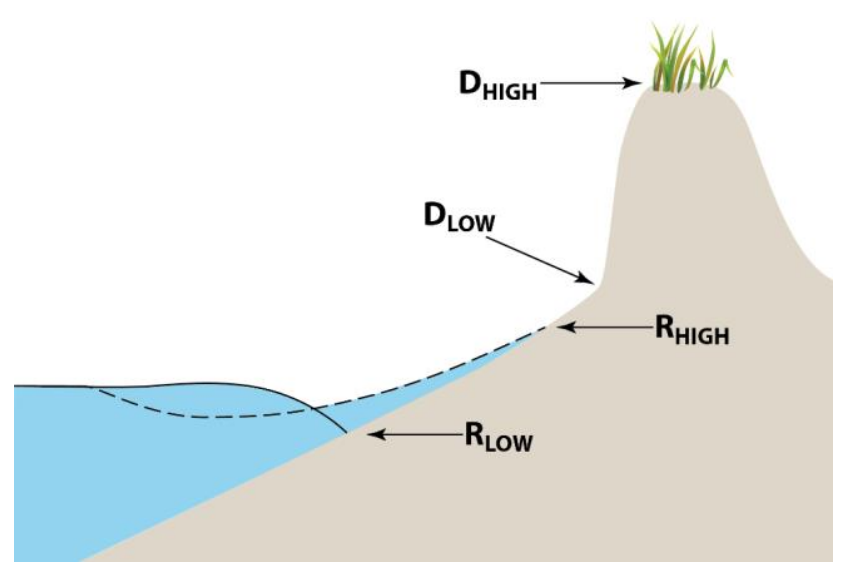

(Sallenger, 2000)
Barrier Island Resiliency

(Houser et al, 2015) 


\section{Padre Island National Seashore (PAIS)}
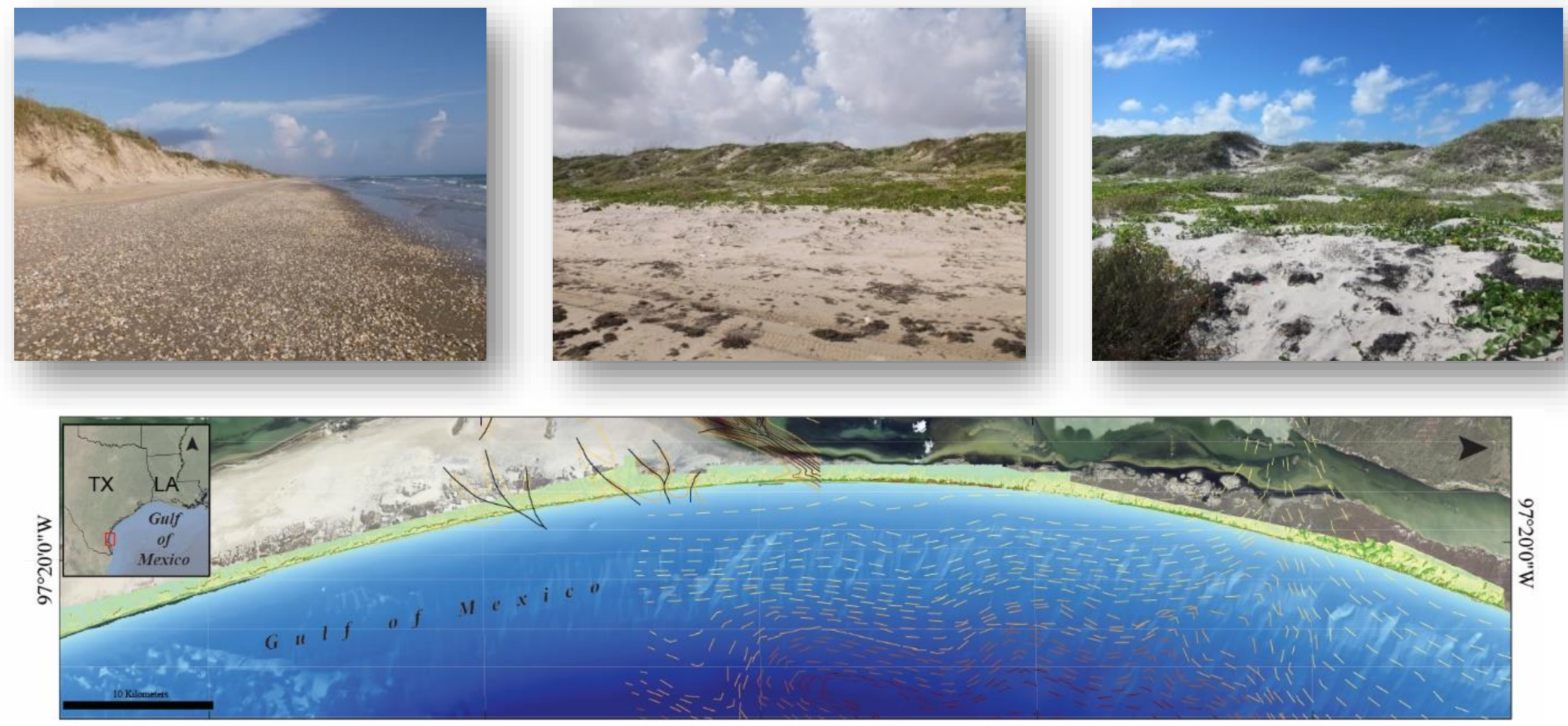

$26^{\circ} 40^{\prime} 0^{\prime \prime} \mathrm{N}$

Fisk (1959) paleosurface contours: Anderson et al (2016) MIS II contours:
$25 \mathrm{ft}$

$14 \mathrm{~m}-24 \mathrm{~m}$ $26^{\circ} 50^{\prime} 0^{\prime \prime} \mathrm{N}$

$50 \mathrm{ft}$

$25 \mathrm{~m}-34 \mathrm{~m}$ $27^{\circ} 0^{\prime} 0^{\prime \prime} \mathrm{N}$

$75 \mathrm{ft}$

$35 \mathrm{~m}-44 \mathrm{~m}$

$$
-100 \mathrm{f}
$$

$-100 \mathrm{ft} \quad-125 \mathrm{ft}$
$-45 \mathrm{~m}-54 \mathrm{~m}--55 \mathrm{~m}-64 \mathrm{~m}$ $27^{\circ} 10^{\prime} 0 " \mathrm{~N}$

Fisk (1959) $27^{\circ} 20^{\prime} 0^{\prime \prime} \mathrm{N}$

Elevation (masl)

$\begin{array}{ccc}-20 & 0 & 15\end{array}$ 


\section{Alongshore Morphometrics}
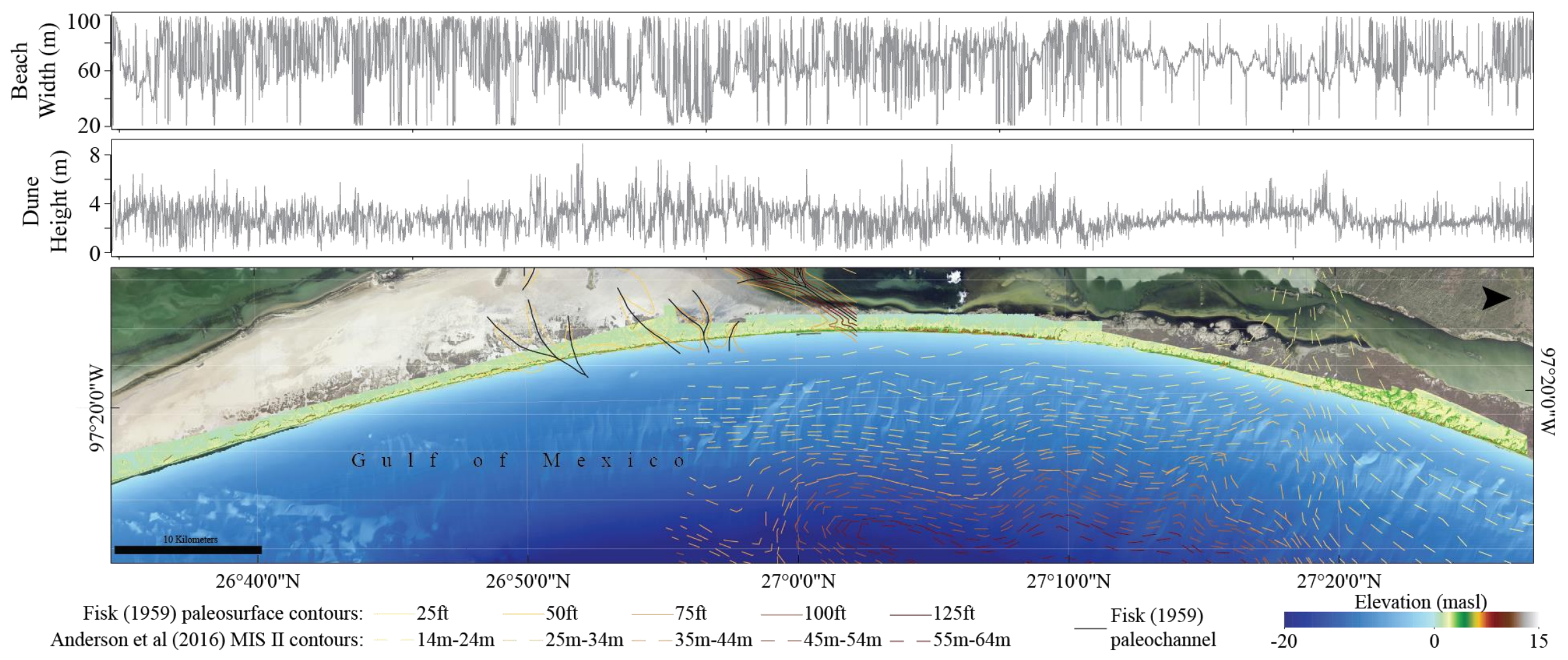

Time-series vs. Spatial-series 


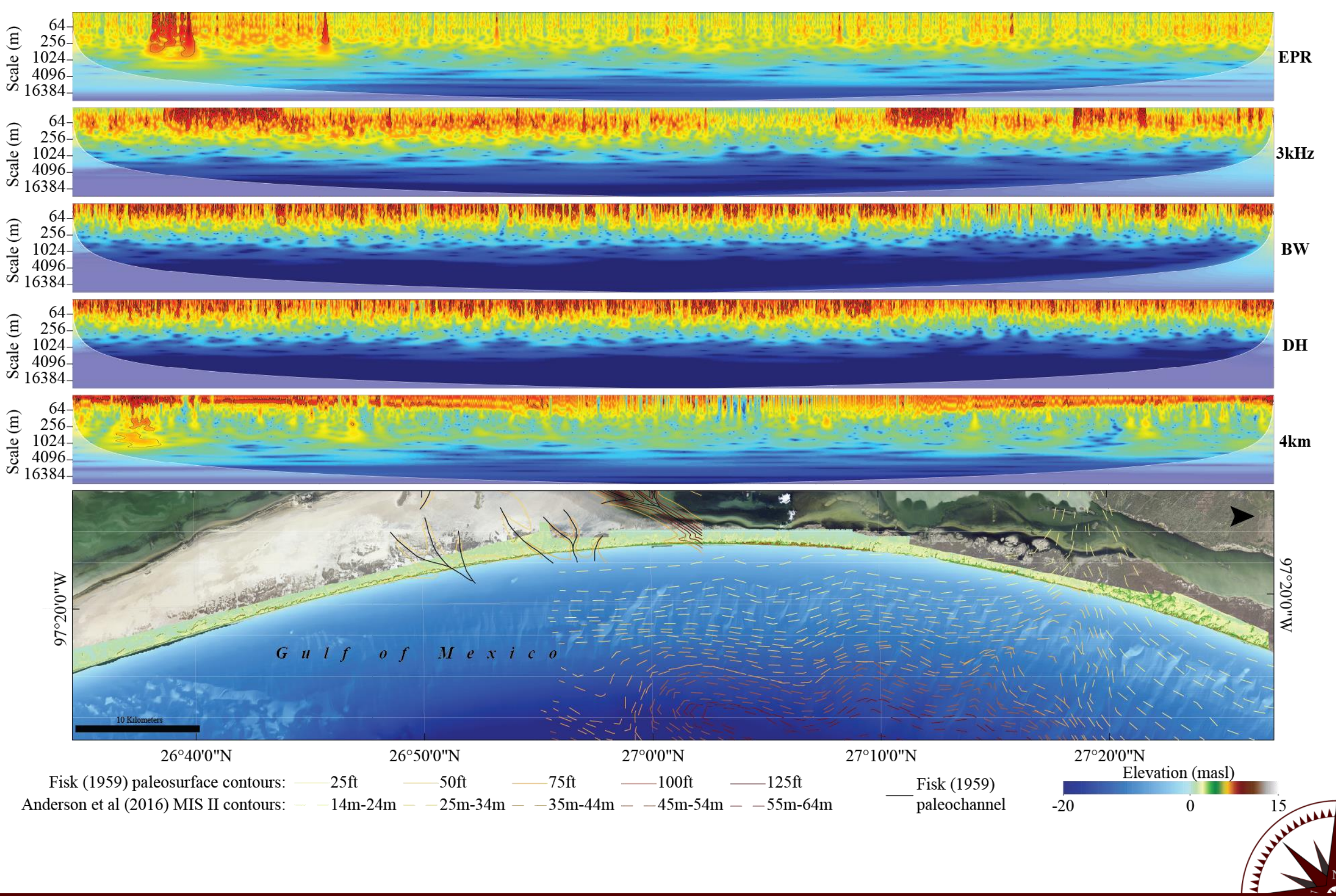

\section{A \begin{tabular}{llllllllll}
\hline & $N$ & $I$ & $V$ & $E$ & $R$ & $S$ & $I$ & $T$ & $Y$
\end{tabular}}

CWT modelled using biwavelet R package (Gouhier et al. 2004) Wernette et al. (in prep) Earth Surface Processes and Landforms 


\section{Peak Spectral Density}

White noise (slope 0)

Pink noise (slope $\sim 1$ )

Red noise (slope $\sim 2$ )

Shoreline change (long-term)

$$
\text { slope }=2.27
$$

Surface morphometrics

$$
\text { slope } \sim 1
$$
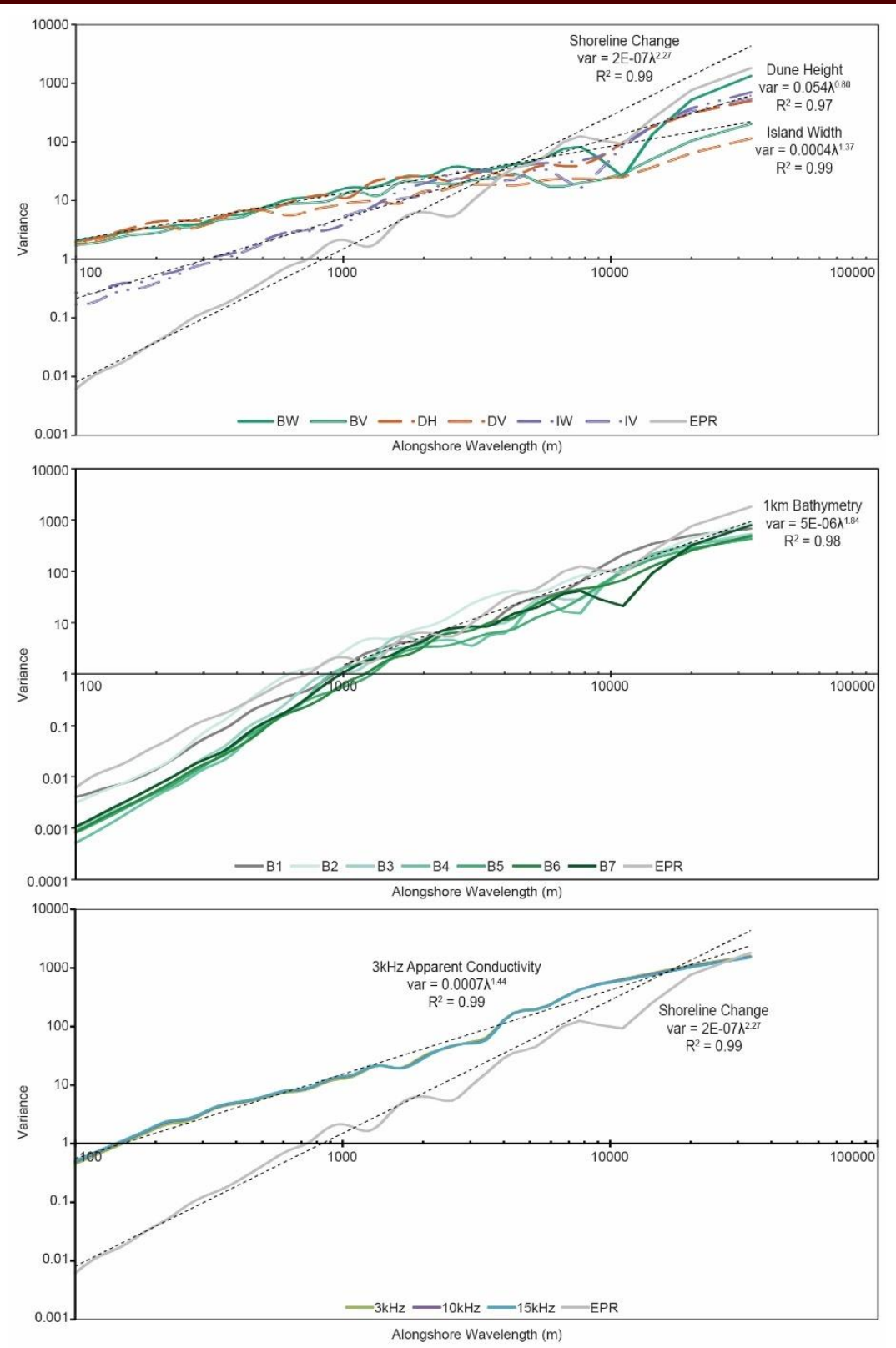


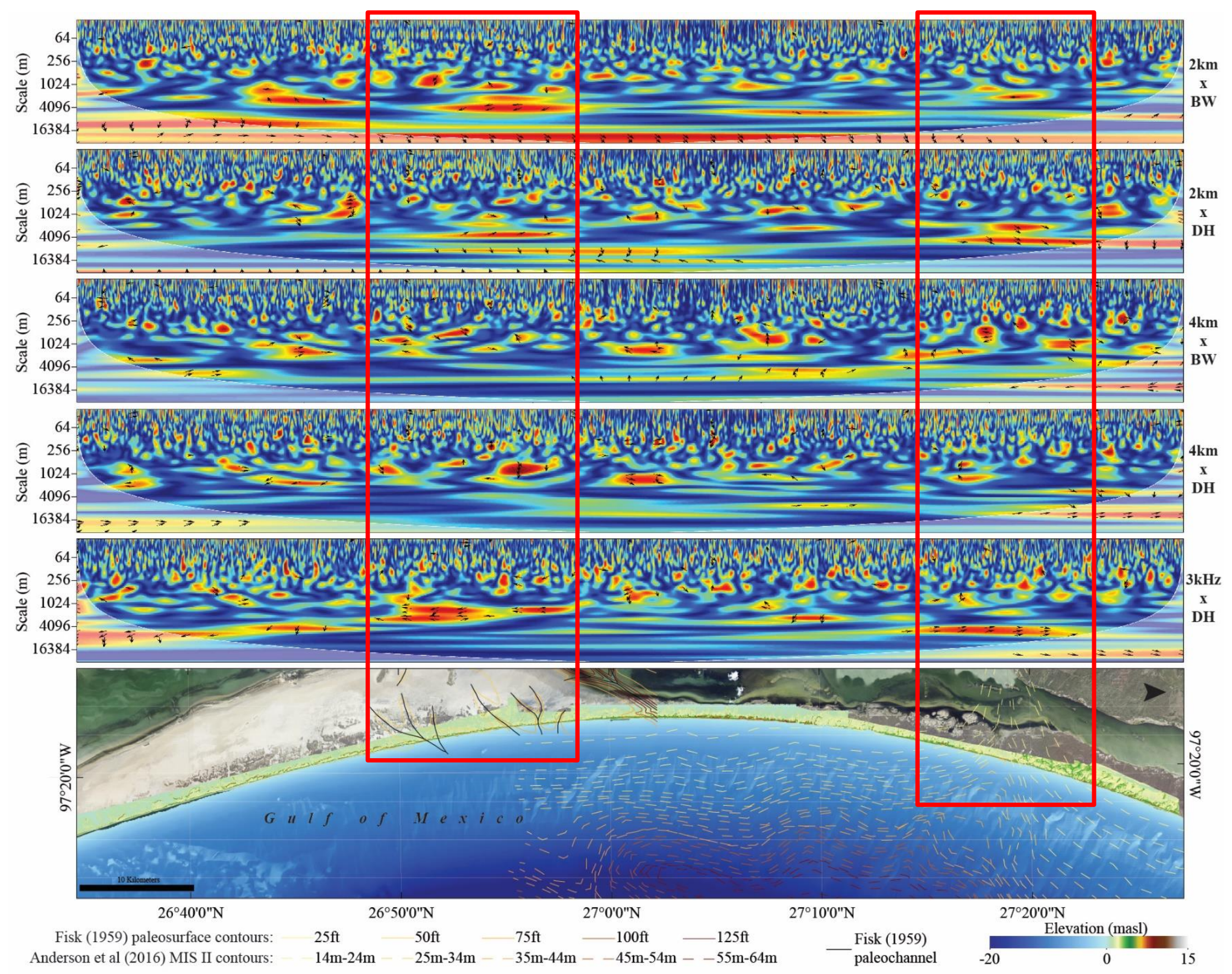

\section{Al] $\begin{array}{lllllllllll} & \mathbf{U} & \mathbf{N} & \mathbf{I} & \mathbf{V} & \mathbf{E} & \mathbf{R} & \mathbf{S} & \mathbf{I} & \mathbf{T} & \mathbf{Y}\end{array}$}

WTC modelled using biwavelet R package (Gouhier et al. 2004) Wernette et al. (in prep) Earth Surface Processes and Landforms 

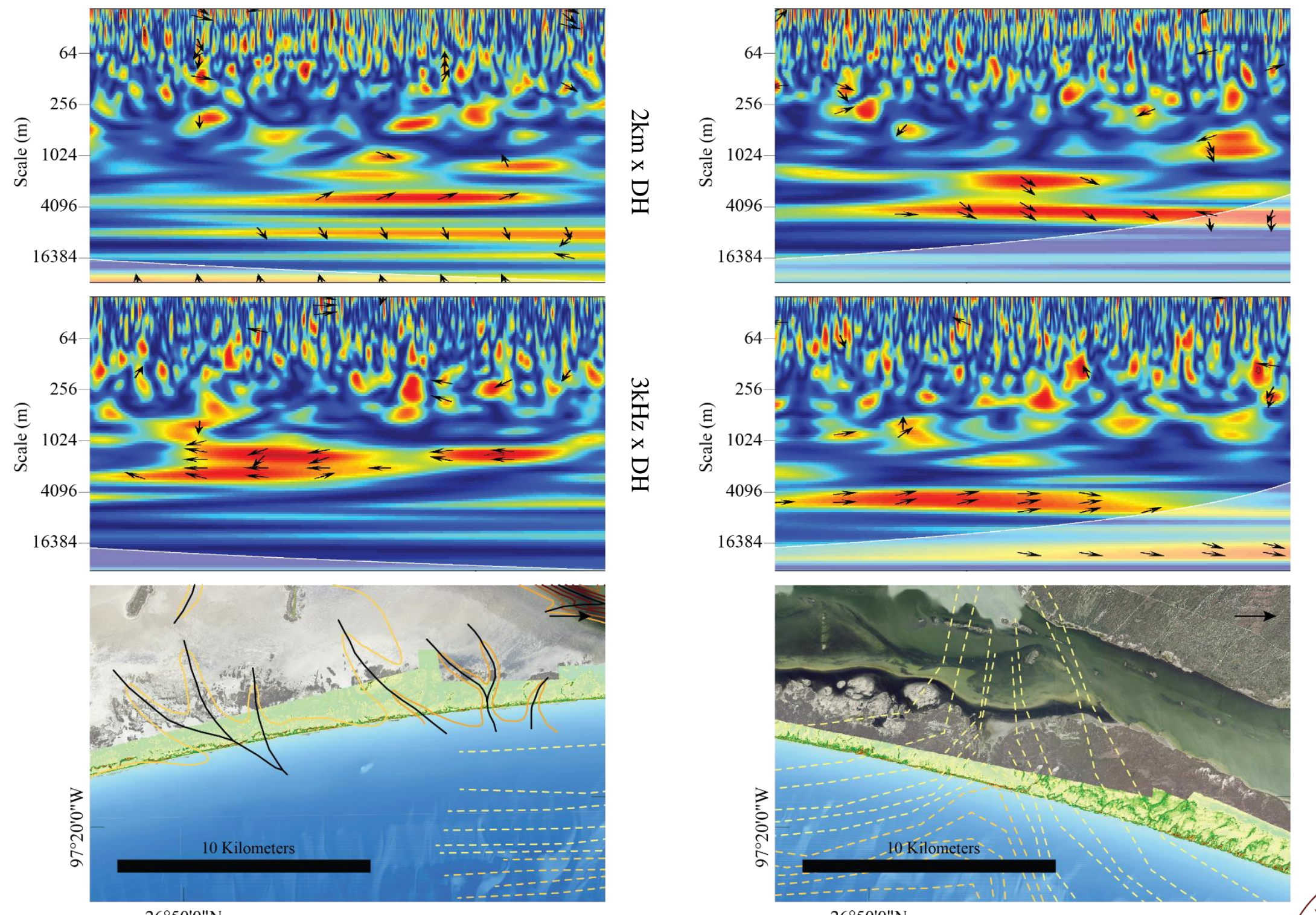


\section{Bicoherence}
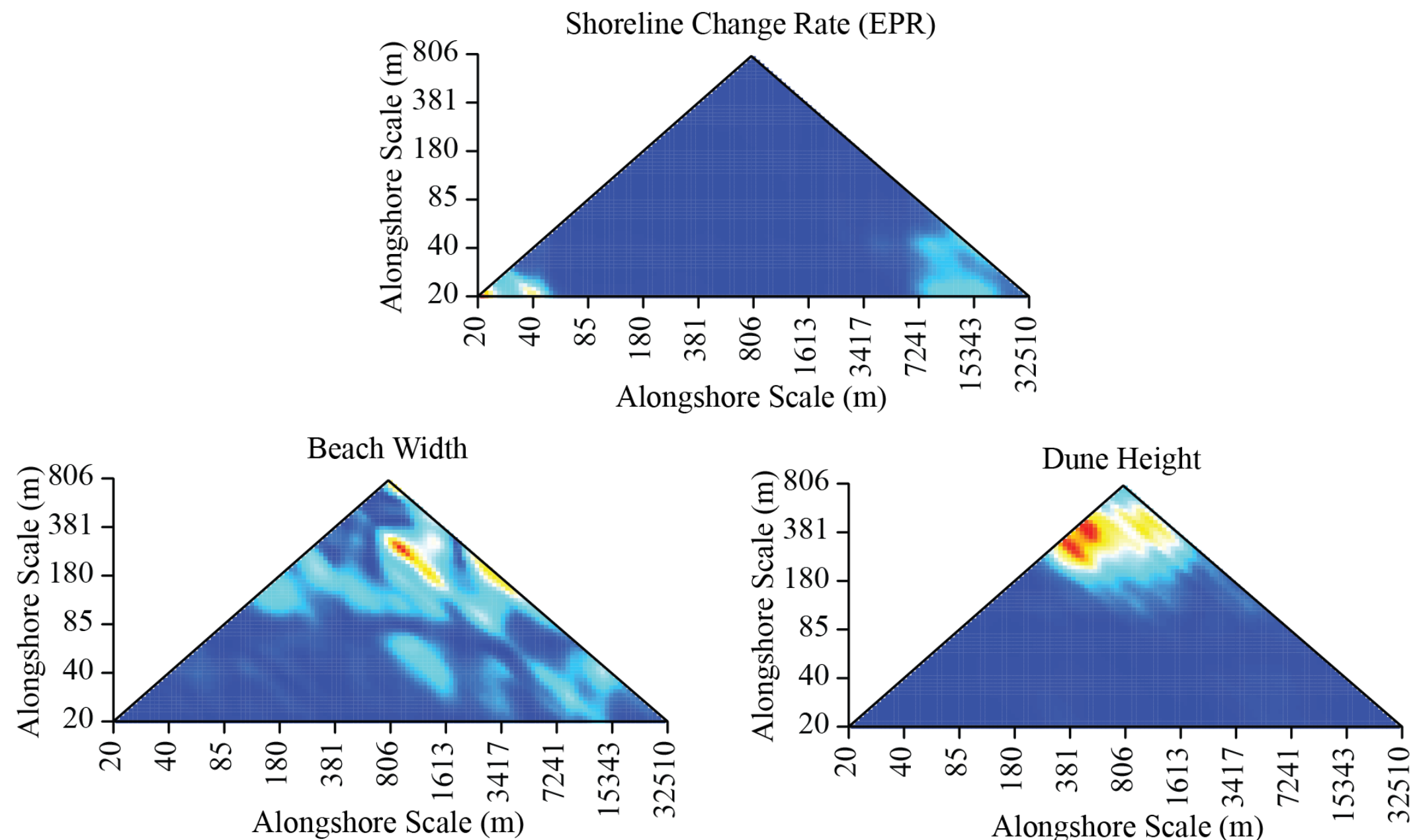


\section{ARFIMA Modelling}

- Autoregressive fractionally-integrated moving average (ARFIMA) (Fraley et al, 2012)

- Evaluate short- and long-range dependencies (SRD and LRD)

$(p, d, q)$ model:

$p \rightarrow$ SRD (autoregressive)

$d \rightarrow$ LRD

$q \rightarrow$ SRD (moving average)

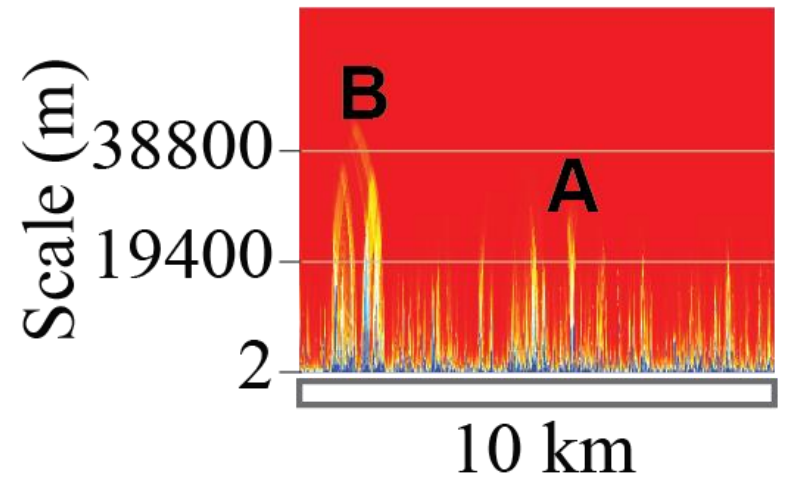



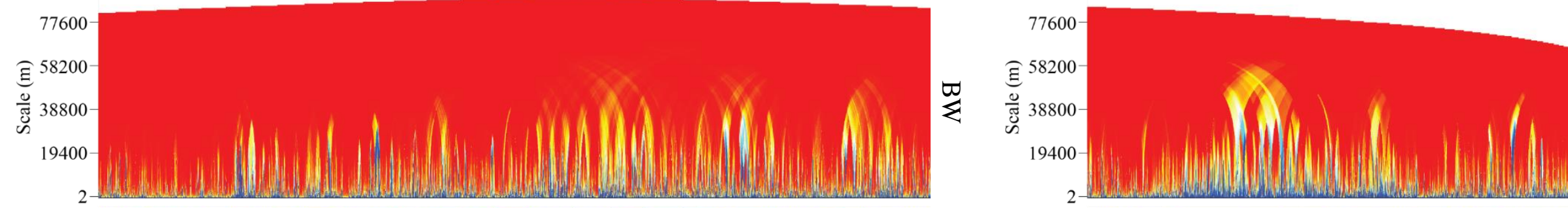

$\underset{\Sigma}{\infty}$
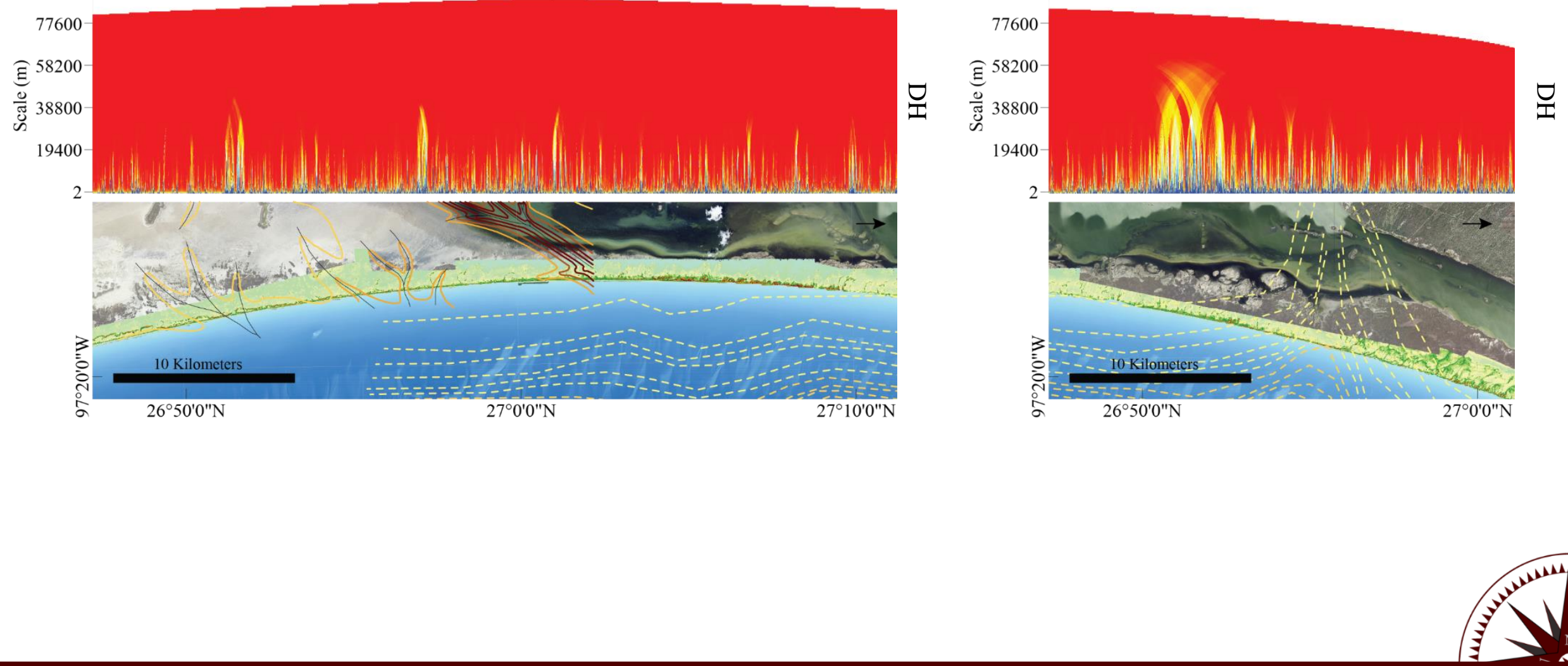

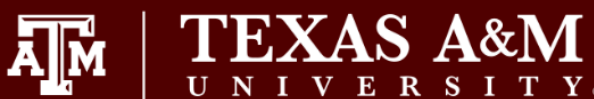

ARFIMA modelled using fracdiff R package (Frayley et al. 2012) Wernette et al. (in prep) Marine Geology 


\section{Directional Dependency}
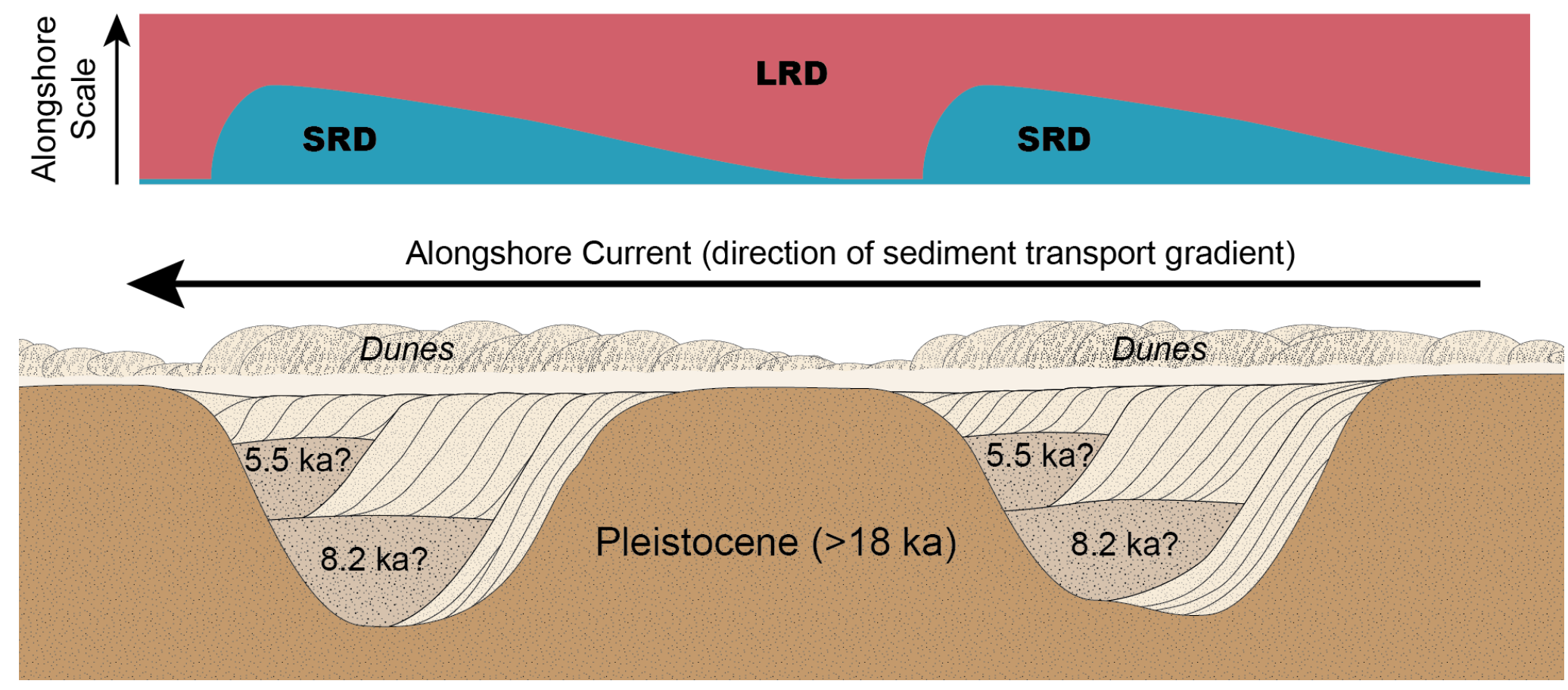


\section{PAIS Development}

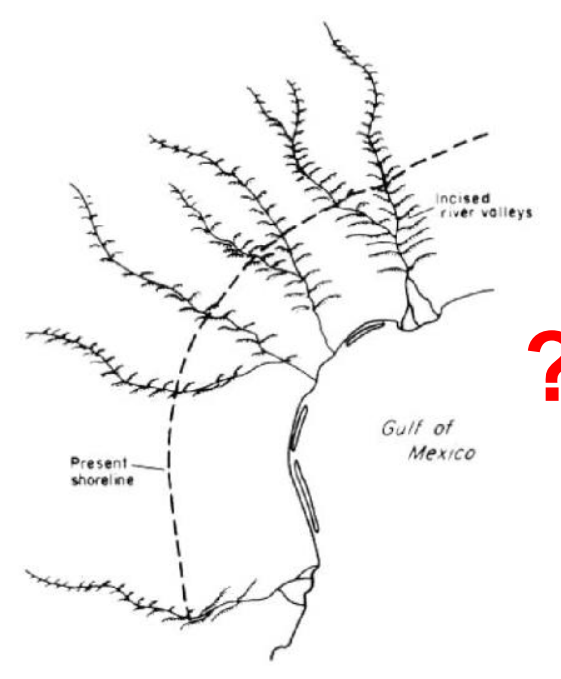

a. 18,000 years ogo

End of Wisconsin Glacial Stage

Sea level -300 to -450 feet

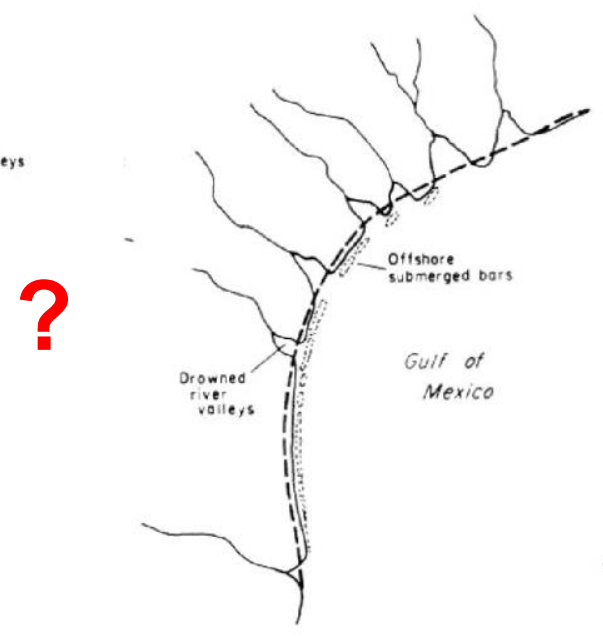

(2) 4,500 years ago

End of Holocene Sea level -15 feet

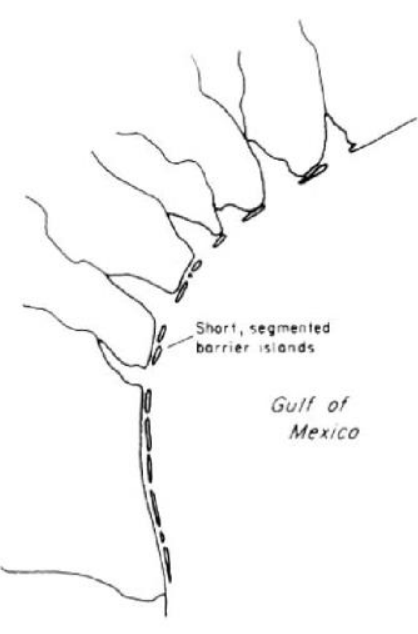

C. 2,800 years ago

Seo level same as present

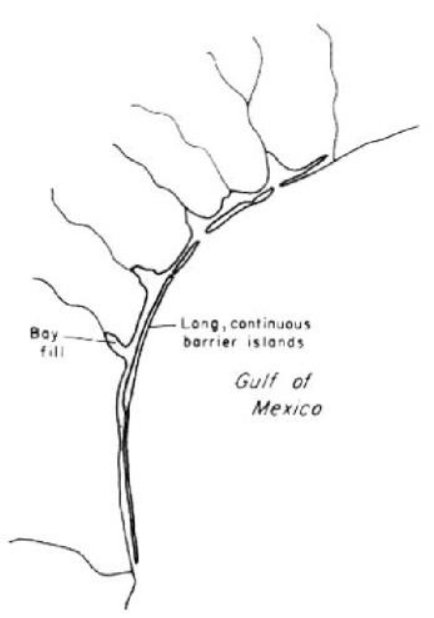

d. Present
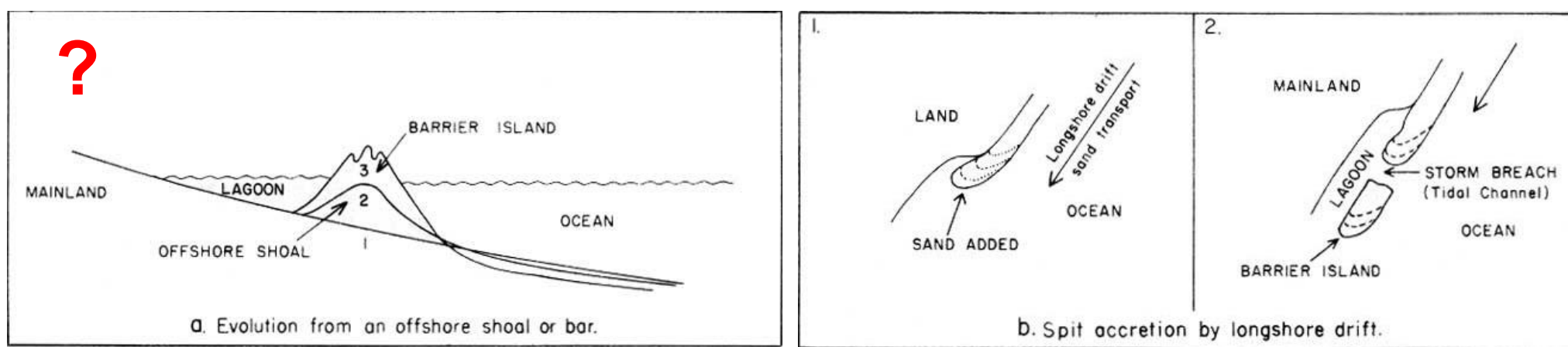

b. Spit occretion by longshore drift.

(Weise and White, 1980)

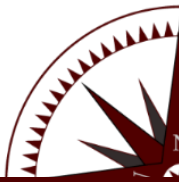

Wernette et al. (in prep) Continental Shelf Research

\section{A]M $\mid \underset{V}{\text { TEXIVAS A\&M }}$}




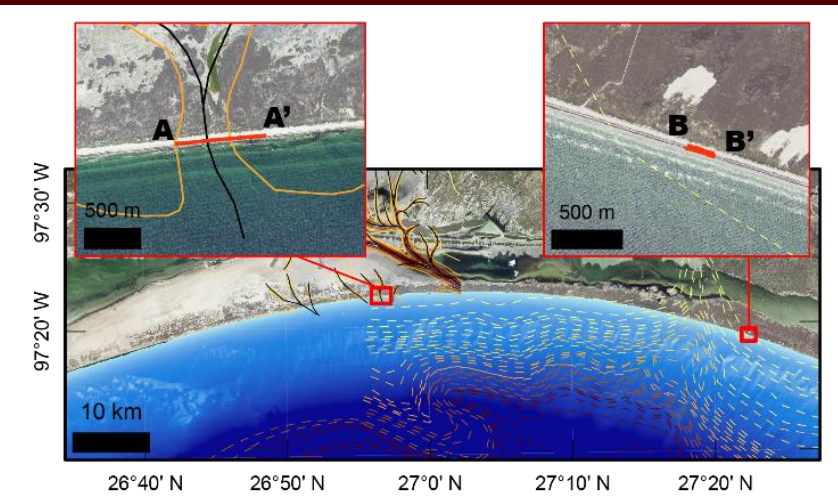

\section{GPR}

B

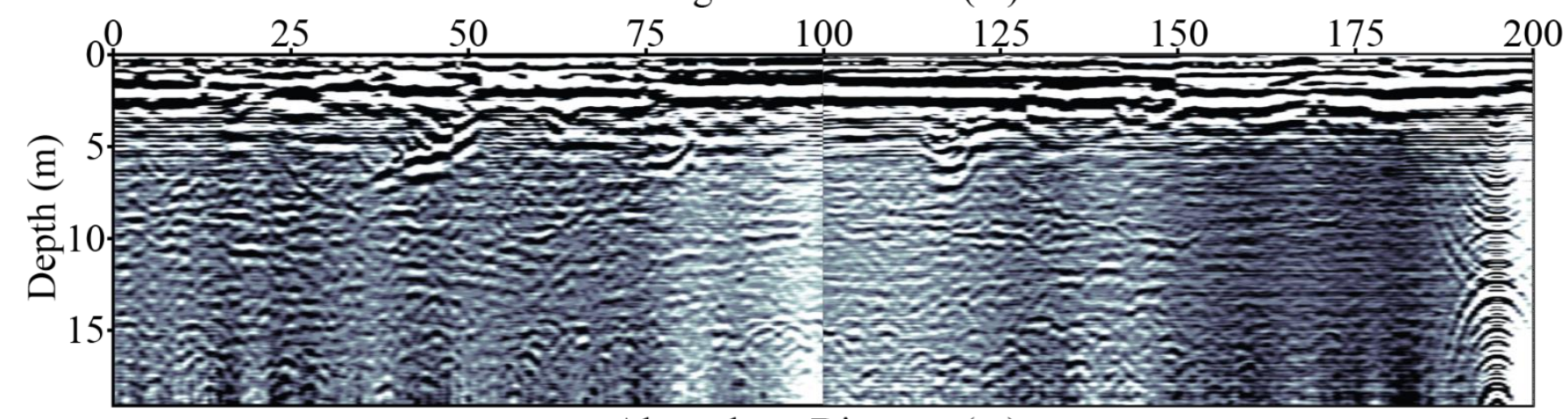

Alongshore Distance (m)

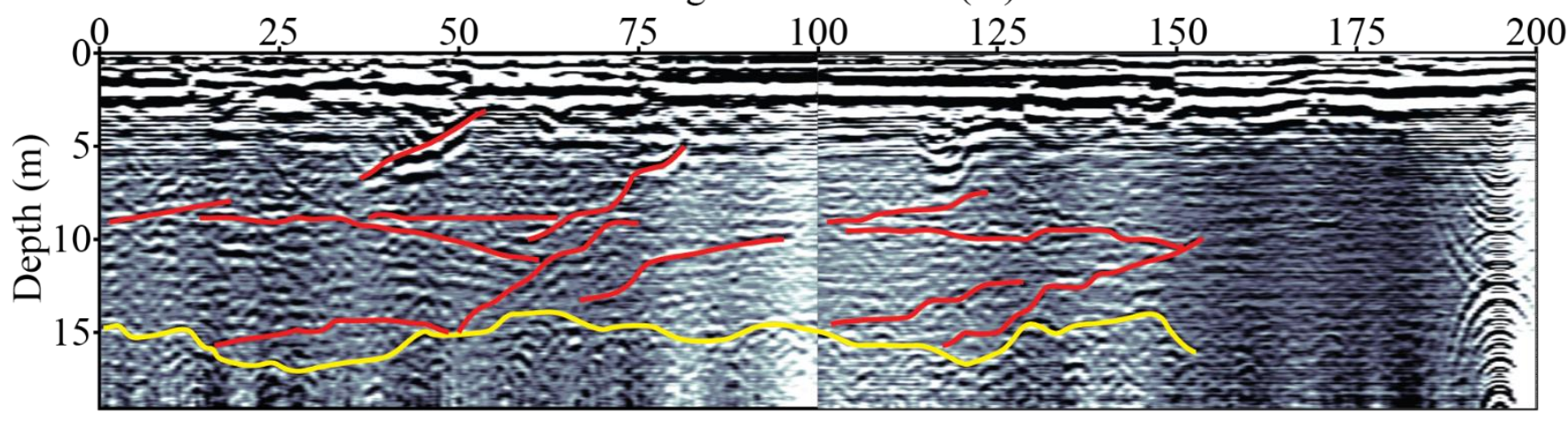

Interpreted geologic structure

MIS II surface?

Wernette et al. (in prep) Continental Shelf Research 


\section{Framework Geology Context}
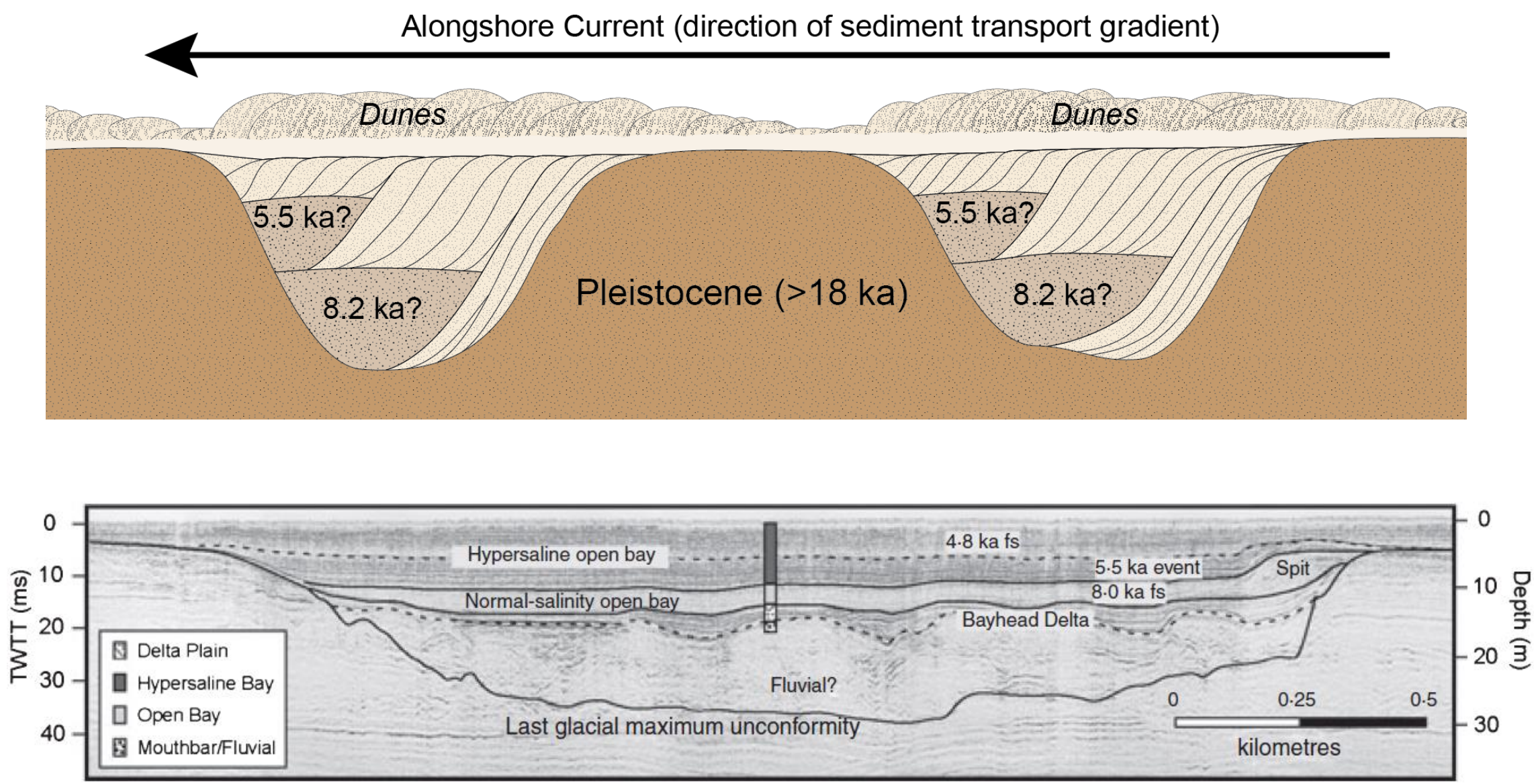

Baffin Bay seismic profile (Simms et al, 2010) 


\section{PAIS Development}

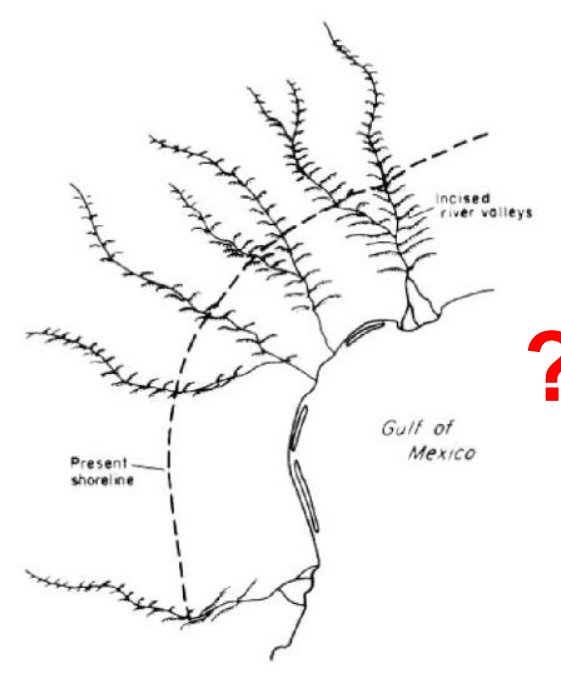

a. 18,000 years ogo

End of Wisconsin Glacial Stage

Sea level -300 to -450 feet

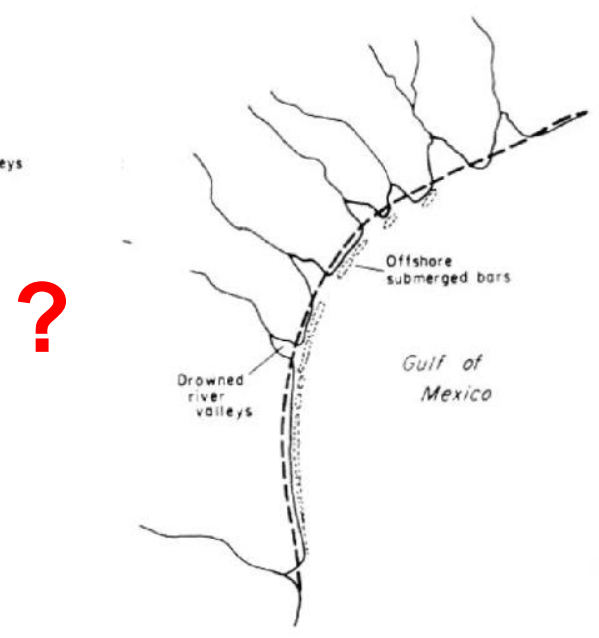

(2) 4,500 years ago

End of Holocene Sea level -15 feet

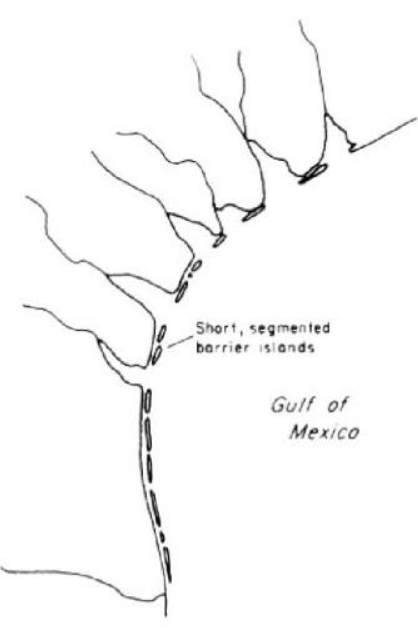

C. 2,800 years ago

Seo level same as present

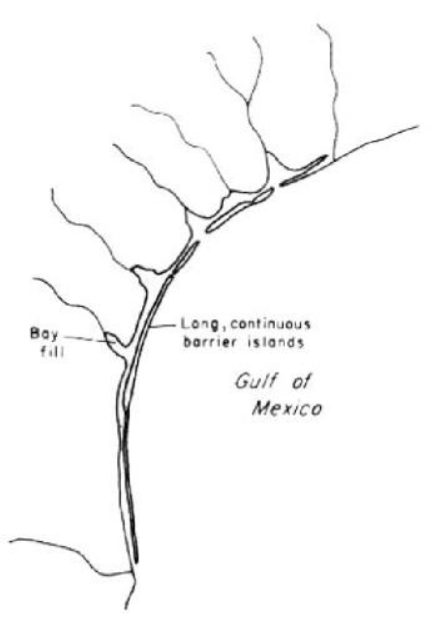

d. Present
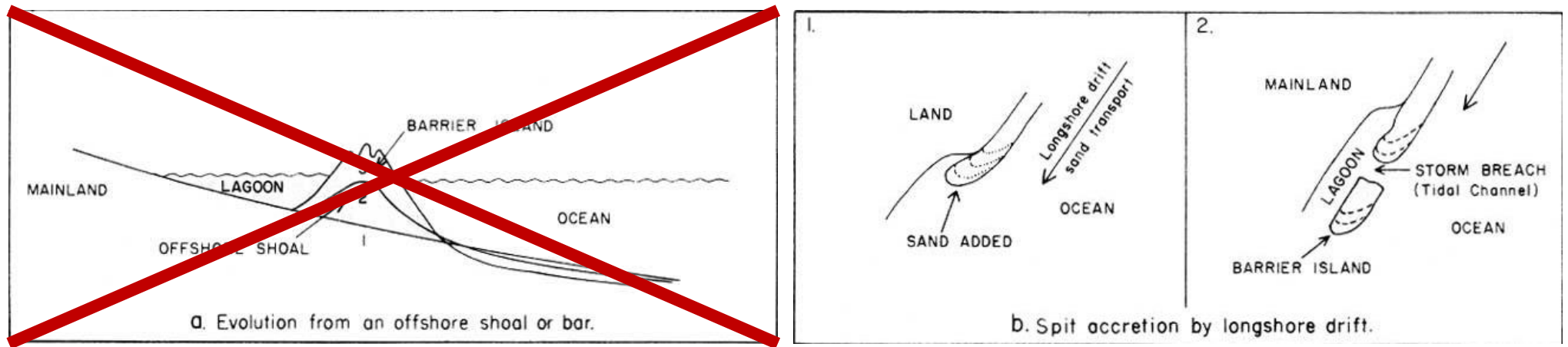

(Weise and White, 1980)

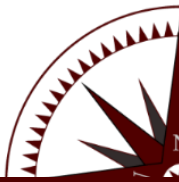

Wernette et al. (in prep) Continental Shelf Research

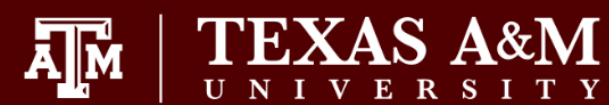




\section{Barrier Island Geomorphology}

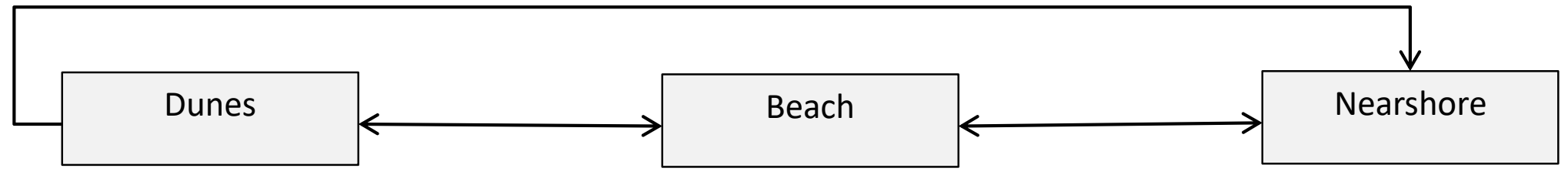

Geologic Framework

- Spatial variations in framework geology affect dune morphology

- Influence barrier island transgression

- Persist through time

- Directional dependencies possible

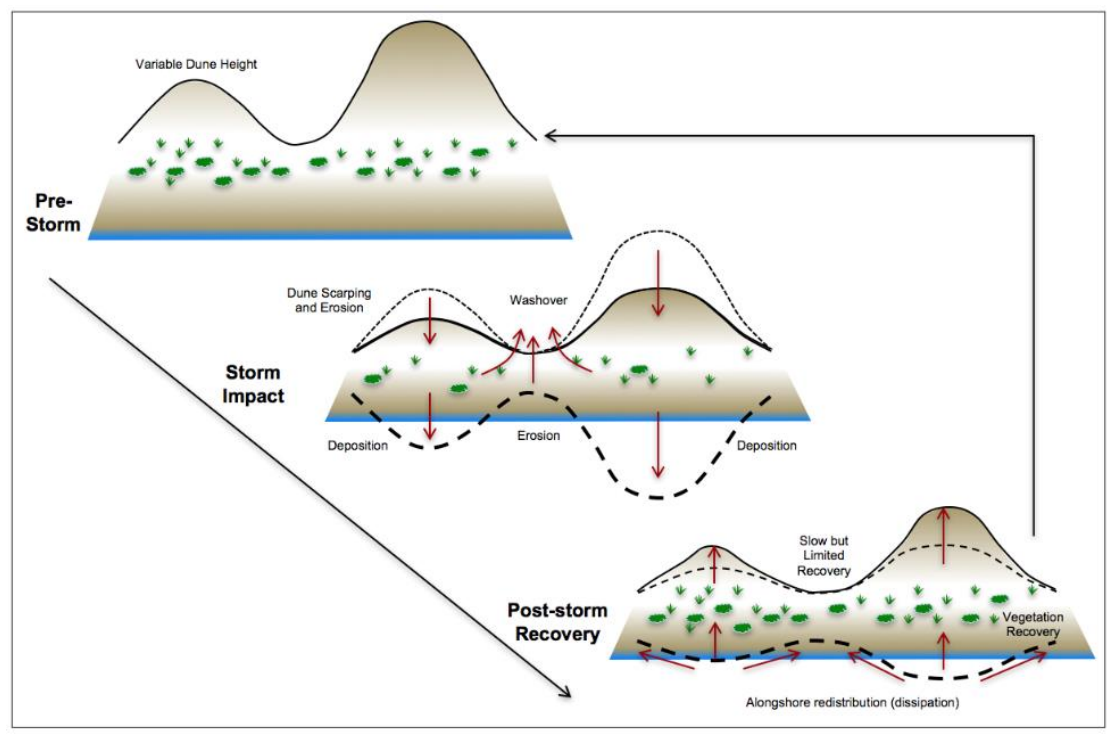

(Houser et al, 2015) 


\section{References}

Anderson, J.B., D.J. Wallace, A.R. Simms, A.B. Rodriguez, R.W.R. Weight, and Z.P. Taha. 2016. Recycling Sediments between Source and Sink During a Eustatic Cycle: Systems of Late Quaternary Northwestern Gulf of Mexico Basin. Earth-Science Reviews 153, 111-138.

Elsayed, M.A.K. 2006a. A Novel Technique in Analyzing Non-Linear Wave-Wave Interaction. Ocean Engineering 33 , 168-180.

-----. 2006b. Wavelet Bicoherence Analysis of Wind-Wave Interaction. Ocean Engineering 33, 458-470.

Fisk, H.N. 1959. Padre Island and Lagunas Madre Flats, Coastal South Texas. Second Coastal Geography Conference, 103-151.

Fraley, C., F. Leisch, M. Maechler, V. Reisen, and A. Lemonte, 2012. Fracdiff: Fractionally Differenced Arima Aka Arfima(P,D,Q) Models, R package version 1.4-2 ed.

Gouhier, T.C., A. Grinstead, and V. Simko, 2016. Biwavelet: Conduct Univariate and Bivariate Wavelet Analyses, 0.20.7 ed.

Houser, C., P. Wernette, E. Rentschlar, H. Jones, B. Hammond, and S. Trimble. 2015. Post-Storm Beach and Dune Recovery: Implications for Barrier Island Resilience. Geomorphology 234, 54-63.

Sallenger, A.H. 2000. Storm Impact Scale for Barrier Islands. Journal of Coastal Research 16, 890-895.

Simms, A.R., N. Aryal, L. Miller, and Y. Yokoyama. 2010. The Incised Valley of Baffin Bay, Texas: A Tale of Two Climates. Sedimentology 57, 642-669.

Weise, B.R. and W.A. White. 1980. Padre Island National Seashore: A Guide to the Geology, Natural Environments, and History of a Texas Barrier Island. Texas Bureau of Economic Geology, 94

Wernette, P., C. Houser, and M.P. Bishop. 2016. An Automated Approach for Extracting Barrier Island Morphology from Digital Elevation Models. Geomorphology 262, 1-7. 


\section{Acknowledgements}

- National Park Service

- James Lindsay, Travis Clapp

- Funding partially provided by

Texas A\&M University College of Geosciences

National Science Foundation

Texas Sea Grant.

- Data was collected under the following National

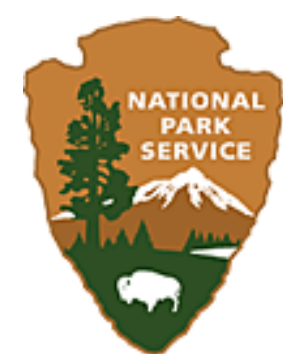

Park Service Research Permits:

\#PAIS-2015-SCI-0004

\#PAIS-2016-SCI-0003

\#PAIS-2016-SCI-0014

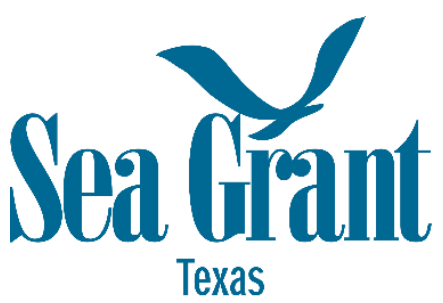

AT TEXAS A\&M UNIVERSITY

TexasSeaGrant.org

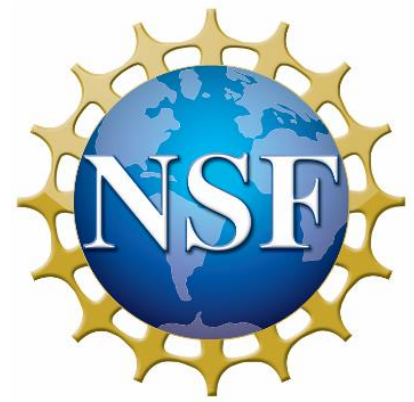




\title{
Assessing the Role of Framework Geology on Barrier Island Geomorphology
}

\author{
Phillipe Wernette ${ }^{1 *}$, Chris Houser ${ }^{2}$, Bradley Weymer ${ }^{3}$, Mark Everett ${ }^{4}$, \\ Michael P. Bishop ${ }^{1}$, and Bobby Reece ${ }^{4}$ \\ ${ }^{1}$ Texas A\&M University, Department of Geography, College Station, TX, USA \\ 2University of Windsor, Department of Earth and Environmental Sciences, Windsor, ON, Canada \\ ${ }^{3}$ GEOMAR Helmholtz Center for Ocean Research Kiel, Kiel, Germany \\ ${ }^{4}$ Texas A\&M University, Department of Geology and Geophysics, College Station, TX, USA
}

